\section{Polyethylene materials with in-chain ketones from nonalternating catalytic copolymerization}

\author{
Maximilian Baur, Fei Lin, Tobias 0. Morgen, Lukas Odenwald, Stefan Mecking*
}

The world's most abundantly manufactured plastic, polyethylene, consists of inert hydrocarbon chains. The introduction of reactive polar groups in these chains could help overcome problematic environmental persistence and enhance compatibility with other materials. We show that phosphinophenolate-coordinated nickel complexes can catalyze nonalternating copolymerization of ethylene with carbon monoxide to incorporate a low density of individual in-chain keto groups in polyethylene chains with high molecular weight while retaining desirable material properties. After processing by conventional injection molding techniques, tensile properties remain on par with those of standard high-density polyethylene while also imparting photodegradability.

P olyethylene is the most abundantly manufactured synthetic polymer, combining facile processing and low-cost production with beneficial mechanical properties (1). The latter arises from crystalline ordering of stretched hydrocarbon chains. This ordering is particularly pronounced for high-density polyethylene (HDPE), which consists of linear chains devoid of branching that would otherwise disturb crystalline packing (2). Polyethylene is hydrophobic and nonpolar and does not easily adhere to polar materials such as metal surfaces or wood. Because of the chemically inert nature of the hydrocarbon chains, polyethylene is not susceptible to degradation reactions and therefore persists when released to the environment.

One prospective approach to overcoming the drawbacks of crystalline polyethylene materials is catalytic copolymerization of ethylene with a low ratio of carbon monoxide. The resulting keto groups in the polyethylene chain could provide an array of desirable modes of reactivity-including photodegradability-as demonstrated for branched low-density polyethylenes (LDPEs) with $\sim 1 \mathrm{~mol} \%$ of keto groups from free-radical high-pressure copolymerization (3-5). Even in linear polyethylene, a low concentration of keto groups is unlikely to disturb the crystalline order (6).

However, such materials have remained elusive, despite numerous efforts toward copolymerization. Compared with copolymerization, alternative post-polymerization oxidation of polyethylene $(7,8)$ requires an additional synthetic step and is not selective for keto functional groups; it also requires problematic reagents. In copolymerization,

University of Konstanz, Department of Chemistry, Universitätsstraße 10, 78457 Konstanz, Germany.

*Corresponding author. Email: stefan.mecking@uni-konstanz.de carbon monoxide exhibits much stronger binding to the catalyst compared with the ethylene monomer, which prevents consecutive incorporation of olefin (9). Thus, rather than a mildly perturbed polyethylene chain, alternating polyketones are formed, which are used in high-melting engineering thermoplastics with entirely different applications and processing properties than polyethylenes (10).

Here, we report that $\mathrm{Ni}$ (II) catalysts can achieve the long-sought nonalternating catalytic copolymerization of ethylene and $\mathrm{CO}$. The resultant materials with desirably low content of keto groups in high-molecular weight polyethylene chains can be melt-processed and feature tensile properties similar to those of commercial HDPE.

For catalyst choice and design, traditional electron-deficient $\mathrm{d}^{0}$-metal sites-such as those used in production of polyolefins (1)-are<smiles></smiles>

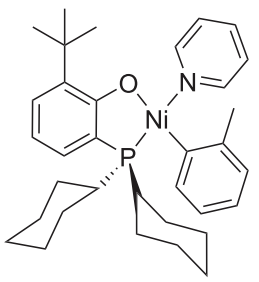

5 2 to 7 .

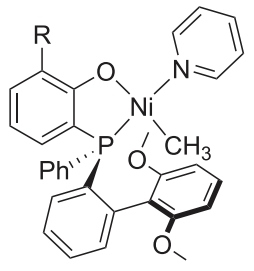

2: $R=$ F $_{F}^{F}$

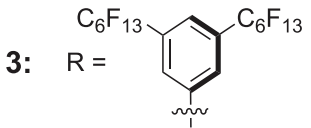

4: $\mathrm{R}=$<smiles>C1CCC2(CC1)CCCC2</smiles>

excluded as they are deactivated by polar molecules such as carbon monoxide. Quenching with carbon monoxide is an established method to deactivate such olefin polymerization catalysts (11). Catalytic processes involving $\mathrm{CO}$, such as olefin carbonylation, generally rely on $\mathrm{d}^{8}$-metal centers (12), and the aforementioned alternating polyketones are produced commercially with cationic palladium catalysts $(10,13)$. Further, the strong binding affinity of CO-widely used as a ligand in organometallic chemistry-must be controlled. Irreversible displacement of other essential ligands, which destroys active sites, must be prevented. In addition, the coordination of $\mathrm{CO}$ or keto groups in the growing chain can potentially block coordination sites required for further chain growth. Notably, the kinetic preference for CO incorporation, which promotes the formation of alternating polyketones, needs to be overcome. In this sense, the target CO copolymerizations are the opposite of catalytic copolymerizations of ethylene with polar vinyl comonomers such as acrylates (14-17), in which the comonomer reactivity can be problematically low.

These arguments prompt consideration of neutral late transition metal catalysts with strongly bound chelating ligands as promising candidates. Compared with more common cationic polymerization catalysts, neutral active sites exhibit a less pronounced preference for binding of $\mathrm{CO}$ versus other ligands, including monomeric olefins. Indeed, the only precedent for consecutive ethylene insertions in CO copolymerization has been observed with neutral phosphinosulfonato palladium catalysts (18-21). However, low-molecular weight brittle waxes with number average

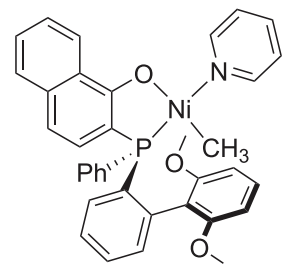

6

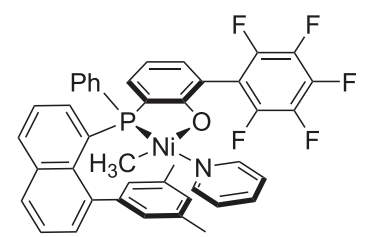

7

Fig. 1. Catalyst precursors studied. Salicylaldiminato complex $\mathbf{1}$, and phosphinophenolato complexes 
molecular weights $M_{\mathrm{n}} \leq 3000 \mathrm{~g} \mathrm{~mol}^{-1}$ (and likely heterogeneous compositions) were formed (22) and no polyethylene plastics could be obtained despite extensive studies by different joint industrial and academic ventures.

Neutral N,O-coordinated salicylaldiminato and P,O-coordinated phosphinophenolato $\mathrm{Ni}$ (II) catalysts in ethylene homopolymerization can produce linear semicrystalline polyethylene with a molecular weight of several million grams per mole (23). High polymerization temperatures are also expected to be favorable for nonalternating chain growth. For the general case of two competing pathways, the preference for the kinetically favored one decreases with increasing temperature according to $k_{a} / k_{b} \sim \exp \left(-\Delta \Delta G^{\nexists} / R T\right)$ assuming a given difference of the energy barriers $\left(\Delta \Delta G^{\neq}\right)$ for both pathways. $R$ is the universal gas constant and $\mathrm{T}$ is temperature. The robust nature of $\mathrm{P}, \mathrm{O}$-coordinated nickel(II) catalysts is advantageous in this regard. They are applied at $140^{\circ} \mathrm{C}$ for industrial ethylene oligomerization (24). However, as a result of their propensity for chain transfer by $\beta$-hydride elimination events, traditional $\mathrm{P}, \mathrm{O}-$ coordinated catalysts yield oligomers or low molecular weight polymers with $M_{\mathrm{n}}<10^{4} \mathrm{~g} \mathrm{~mol}^{-1}$, even at lower reaction temperatures $(25-27)$. Only recently has this long-prevalent perspective been revised by the finding that appropriately bulky substituents can strongly suppress chain transfer to afford high molecular weight polyethylenes $(28,29)$.

Notwithstanding the literature consensus that such catalysts are rapidly deactivated by $\mathrm{CO}$ and at most form only alternating polyketones with low activity $(26,30)$, we exposed state-of-the-art salicylaldiminato (1) and phosphinophenolato (2) (Fig. 1) catalyst precursors to ethylene (E)/CO mixtures with low partial pressures of $\mathrm{CO}$ (Table 1 ) at polymerization temperatures found to be optimum in the established ethylene polymerizations with these catalysts. With (1), only small amounts of polymer were obtained even at an E/CO ratio of $>10^{2}$ (table $\mathrm{S} 3$, entries 11 and 12 ). Infrared (IR) spectra of the polymer were indicative of an alternating polyketone. By contrast, with (2), substantial amounts [up to 4 grams versus $\sim 60 \mathrm{mg}$ with (1)] of a polymer were obtained with a carbonyl band at a distinctly different wavelength than that of alternating polyketones (Table 1, entries 1 to 5 , and Fig. 2B).

Encouraged by these findings, we explored a series of custom-modified, state-of-the-art neutral P,O-chelated catalyst precursors with bulky substituents on the phenolate moiety as well as aryl groups shielding one or both apical positions (Fig. 1; for synthesis details and full characterization data, see supplementary materials). Catalyst precursors (2) through

A
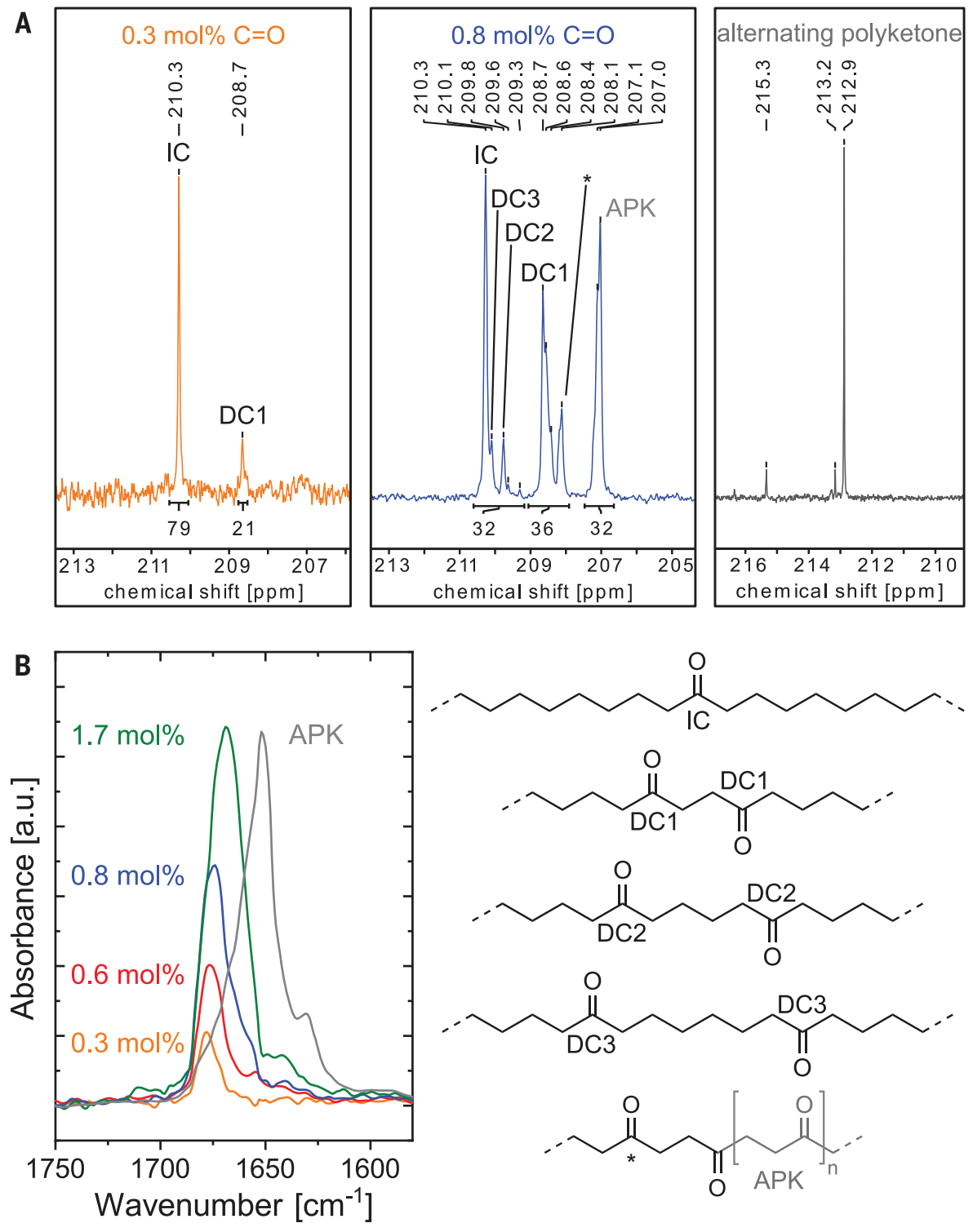<smiles>CCCCCOC(=O)C(=O)CCCCCCC(=O)CCCC</smiles><smiles>CCCC(=O)CCC(=O)CCC(=O)CC</smiles>

Fig. 2. Microstructure characterization of copolymers. (A) Carbonyl regime of ${ }^{13} \mathrm{C}$ NMR spectra of ${ }^{13} \mathrm{CO}$ copolymers with different keto contents $\left(\mathrm{C}_{2} \mathrm{D}_{2} \mathrm{Cl}_{4}\right.$ solvent, $\left.383 \mathrm{~K}\right)$ and an alternating polyketone (hexafluoroisopropanol $/ \mathrm{C}_{6} \mathrm{D}_{6}$ solvent, $300 \mathrm{~K}$ ) for comparison. IC, Isolated carbonyl; DCX, Double carbonyl separated by $X$ repeat units of ethylene; APK, Alternating polyketone; *, Carbonyl adjacent to an alternating sequence. (B) IR spectra of copolymers with different keto content, and alternating polyketone for comparison. The absorbance intensity of alternating polyketones is adjusted for clarity. Note these are ${ }^{13} \mathrm{CO}$ copolymers as in (A), which shifts the absorption frequency compared with nonlabeled copolymers (See figs. S25 and S26 for the IR spectra of the latter).

(6) were all active in the presence of $\mathrm{CO}$ and, at high $\mathrm{E} / \mathrm{CO}$ ratios on the order of 100 , formed polymers that were nonalternating polyketones on the basis of IR spectral characterization (Table 1 and table S3).

The microstructures of the polymers were elucidated by ${ }^{13} \mathrm{C}$ nuclear magnetic resonance (NMR) spectroscopic methods. To enhance sensitivity, copolymers from pressure reactor experiments with ${ }^{13} \mathrm{CO}$ were used. By a combination of $1 \mathrm{D}$ and 2D NMR methods and reference to literature data, ${ }^{1} \mathrm{H}$ and ${ }^{13} \mathrm{C}$ NMR spectra could be assigned fully (figs. S16 to S18). This analysis revealed the presence of isolated ketone groups in the polyethylene chain (Fig. 2A). Further, nonalternating motifs of ketone groups in proximate positions to each other along the chain could be differentiated, and alternating motifs were observable. At desirable values of $\sim 1 \% \mathrm{CO}$ incorporation, most keto groups were nonalternating, and isolated keto groups were prevalent. The overall 
Table 1. Polymerization experiments.*

\begin{tabular}{|c|c|c|c|c|c|c|c|c|c|c|c|}
\hline Entry & Cat. & p [bar] & $t[\mathrm{~min}]$ & $\mathrm{CO}$ in feed [mol \%] ${ }^{\dagger}$ & Yield [g] & TOF $^{\ddagger}$ & $\begin{array}{c}\chi^{\S} \\
{[\mathrm{mol} \%]}\end{array}$ & $\begin{array}{l}\text { I / NA / A" } \\
\text { [mol \%] }\end{array}$ & $\begin{array}{c}M_{\mathrm{n}}^{\#} \\
{\left[10^{3} \mathrm{~g} \mathrm{~mol}^{-1}\right]}\end{array}$ & $M_{\mathrm{w}} / M_{\mathrm{n}}^{\#}$ & $\begin{array}{c}T_{\mathrm{m}}\left[{ }^{\circ} \mathrm{C}\right] \\
\text { (\%cryst.) }^{* *}\end{array}$ \\
\hline 1 & 2 & 5 & 10 & 0.2 & 0.80 & 15.6 & $0.3(0.4)$ & $69 / 11 / 20$ & 54 & 1.7 & $133(71)$ \\
\hline 2 & 2 & 5 & 60 & 0.2 & 2.07 & 7.41 & $0.3(0.4)$ & $70 / 22 / 8$ & 102 & 1.5 & $135(66)$ \\
\hline 3 & 2 & 5 & 120 & 0.2 & 4.26 & 7.61 & $0.3(0.3)$ & $79 / 21 / 0$ & 140 & 1.6 & $134(68)$ \\
\hline 4 & 2 & 5 & 120 & 0.6 & 1.98 & 3.53 & $0.8(1.1)$ & $32 / 36 / 32$ & 147 & 1.8 & $135(62)$ \\
\hline 5 & 2 & 10 & 240 & 0.6 & 3.31 & 2.95 & $0.6(1.0)$ & $43 / 33 / 24$ & 216 & 1.8 & $136(60)$ \\
\hline 6 & 3 & 5 & 120 & 0.6 & 1.13 & 2.02 & $1.0(1.4)$ & $21 / 29 / 49$ & 60 & 1.7 & $133(69)$ \\
\hline 7 & 4 & 5 & 120 & 0.6 & 0.26 & 0.46 & $3.7(4.4)$ & $28 / 34 / 38$ & 84 & 1.7 & $134(63)$ \\
\hline 8 & 5 & 5 & 120 & 0.6 & 0.44 & 0.79 & $1.6(3.0)$ & $7 / 34 / 59$ & 45 & 1.6 & $133(69)$ \\
\hline 9 & 6 & 5 & 120 & 0.6 & 0.82 & 1.47 & $1.1(2.5)$ & $18 / 33 / 49$ & 90 & 1.7 & $135(67)$ \\
\hline
\end{tabular}

*Reaction conditions: $10 \mu \mathrm{mol}$ precatalyst loading, $100^{\circ} \mathrm{C}, 100 \mathrm{ml}$ of toluene, $500 \mathrm{rpm}$ pitched blade stirrer. $\quad$ †Carbon monoxide content in the feed gas. $\quad$ †TOF given in units of $10^{3} \mathrm{~mol}$ $\left[\mathrm{C}_{2} \mathrm{H}_{4}\right] \mathrm{mol}^{-1}[\mathrm{Ni}] \mathrm{h}^{-1}$. \&Carbon monoxide incorporation determined by IR spectroscopy. In brackets: Carbon monoxide incorporation calculated from ${ }^{13} \mathrm{C}$ NMR spectroscopy by integration of the ${ }^{13} \mathrm{C}$ labeled $\mathrm{C}=0$ signals in relation to the overall integral, considering natural abundance of ${ }^{13} \mathrm{C}$ versus ${ }^{12} \mathrm{C}$. शl: isolated carbonyls, NA: nonalternating adjacent carbonyls (See Fig. 2 for specification). A, alternating polyketone segments. Determined by ${ }^{13} \mathrm{C}$ NMR spectroscopy. \#Determined by GPC in 1,2-dichlorobenzene at $160^{\circ} \mathrm{C}$ through universal calibration versus polystyrene standards. $\quad{ }^{* *}$ Determined by DSC, second heating cycle.

keto content was determined by quantitative NMR spectroscopy and confirmed by IR spectroscopy (Table 1, Fig. 2B, and figs. S25 and S26). As anticipated, these in-chain keto motifs do not compromise the desirable material properties of polyethylene. Wide angle x-ray scattering (WAXS) diffractograms of the materials are virtually identical to that of HDPE (Fig. 3A), underlining a conserved solid-state structure. Also, melting and crystallization points are virtually unaltered (Fig. $3 \mathrm{C})$. Most notably, the keto-modified polyethylenes possess high molecular weights [up to weight-average molecular weight $\left(M_{\mathrm{w}}\right)$ $\left.\sim 400,000 \mathrm{~g} \mathrm{~mol}^{-1}, M_{\mathrm{n}} \sim 220,000 \mathrm{~g} \mathrm{~mol}^{-1}\right]$. This result is notable in view of the very low molecular weights observed in ethylene-CO copolymerizations yielding any partially nonalternating motifs to date (22), and it is a crucial factor underlying useful mechanical properties. Indeed, tensile tests on injection molded test bars showed a ductile behavior comparable to commercial HDPE (Fig. 3D): modulus of elasticity $E=1062 \pm 53 \mathrm{MPa}$ and tensile strength $\sigma_{\mathrm{y}}=26.7 \pm 0.2 \mathrm{MPa}$, compared with literature values of $E=900 \mathrm{MPa}$ and $\sigma_{\mathrm{y}}=27 \mathrm{MPa}$ for HDPE, and $E=240 \mathrm{MPa}$ and $\sigma_{\mathrm{y}}=12 \mathrm{MPa}$ for LDPE (31). No evidence for undesirable cross-linking that could hamper melt processing was observed; crosslinking promoted by keto motifs has previously been an issue in the thermoplastic processing of commercial polyketone resins (32). Exposure of keto-PE films to simulated sunlight confirmed their photodegradability. After irradiation of films floating on a water bath with a light intensity corresponding to $\sim 5$ months of natural sunlight in Southern Europe, the onset of degradation was evidenced by embrittlement and me- chanical disintegration of the previously ductile samples, and manifested by an observable weight loss of several weight percent. IR spectroscopy on a ${ }^{13} \mathrm{CO}$-labeled sample showed that in these short-term degradation experiments the keto groups were only partially consumed; in addition, new keto and ester groups formed, which can enable further chain degradation beyond the observed interval. By contrast, a reference sample of keto-free ethylene homopolymer remained unaltered (see figs. S32 to S34 for detailed data).

At given initial reaction conditions, the composition of the resultant polymer did not vary considerably with increasing reaction time or increasing polymer yield (Table 1 , entries 1 to 3 and 5 and table S3, entries 15 and 16). A homogeneous material was consistently formed, opposed to perturbation of microstructure and composition as a result of a conceivable alteration of the relative concentrations of the monomers. Compared with ethylene homopolymerization, the copolymerization yields and average activities were lowered (Table 1, entry 1, versus table S4, entry 2; see table S4 for further homopolymerization data). However, this is not because of a problematically rapid catalyst deactivation. Catalysts were stable for hours in CO copolymerizations (Table 1 , entries 1 to 5). Presumably, in addition to $\mathrm{CO}$ binding, reversible chelating binding of formed keto groups slows down chain growth to a certain extent as concluded from the observation of overall increased productivities with increasing monomer concentration at a given $\mathrm{E} / \mathrm{CO}$ ratio (Table 1, entries 1 and 4, versus table S3, entries 17 and 16; see fig. S35 for a scheme of reversible deactivation pathways). A thresh- old polymerization reaction temperature is required to overcome this retardation. Thus, efficient copolymerization with (2) requires ca. $100^{\circ} \mathrm{C}$, whereas homopolymerizations can proceed rapidly at lower temperatures (table S4, entries 1, 4 to 8). As anticipated, higher polymerization reaction temperatures $\left(>70^{\circ} \mathrm{C}\right)$ also decrease the $\mathrm{CO}$ incorporation and in fact are necessary to achieve nonalternation (Table 1, entries 1 to 5, and table S3 entry 17, versus table S3, entry 18). This observation underlines the crucial role of the temperature stability of catalysts $(\mathbf{2})$ to $(\mathbf{6})$ in enabling the nonalternating copolymerizations of keto-modified polyethylenes. Beyond this, the underlying phosphinophenolate motif also appears particularly well suited to nonalternating copolymerization, with catalyst (2) standing out in detail in terms of its combination of productivity, microstructure and produced polymer molecular weight. By comparison, catalyst (1)-even at an elevated polymerization temperature-formed only alternating polyketones (table $\mathrm{S} 3$, entry 12 ). This privileged nature of the phosphinophenolate motif may be related to the strong metalphosphine bond, which can counteract irreversible deactivation by ligand displacement and reduce the preference for $\mathrm{CO}$ versus ethylene incorporation by enhancing the electron density at the metal.

The high molecular weights of the ketomodified polyethylenes suggest that the presence and incorporation of $\mathrm{CO}$ does not promote any problematic chain transfer reactions. Indeed, an analysis of the polymers' endgroups shows only those types of endgroups also found in homopolyethylenes, namely olefinic endgroups originating from $ß$-hydride transfer of the Ni-polymeryl species (fig. S16). 

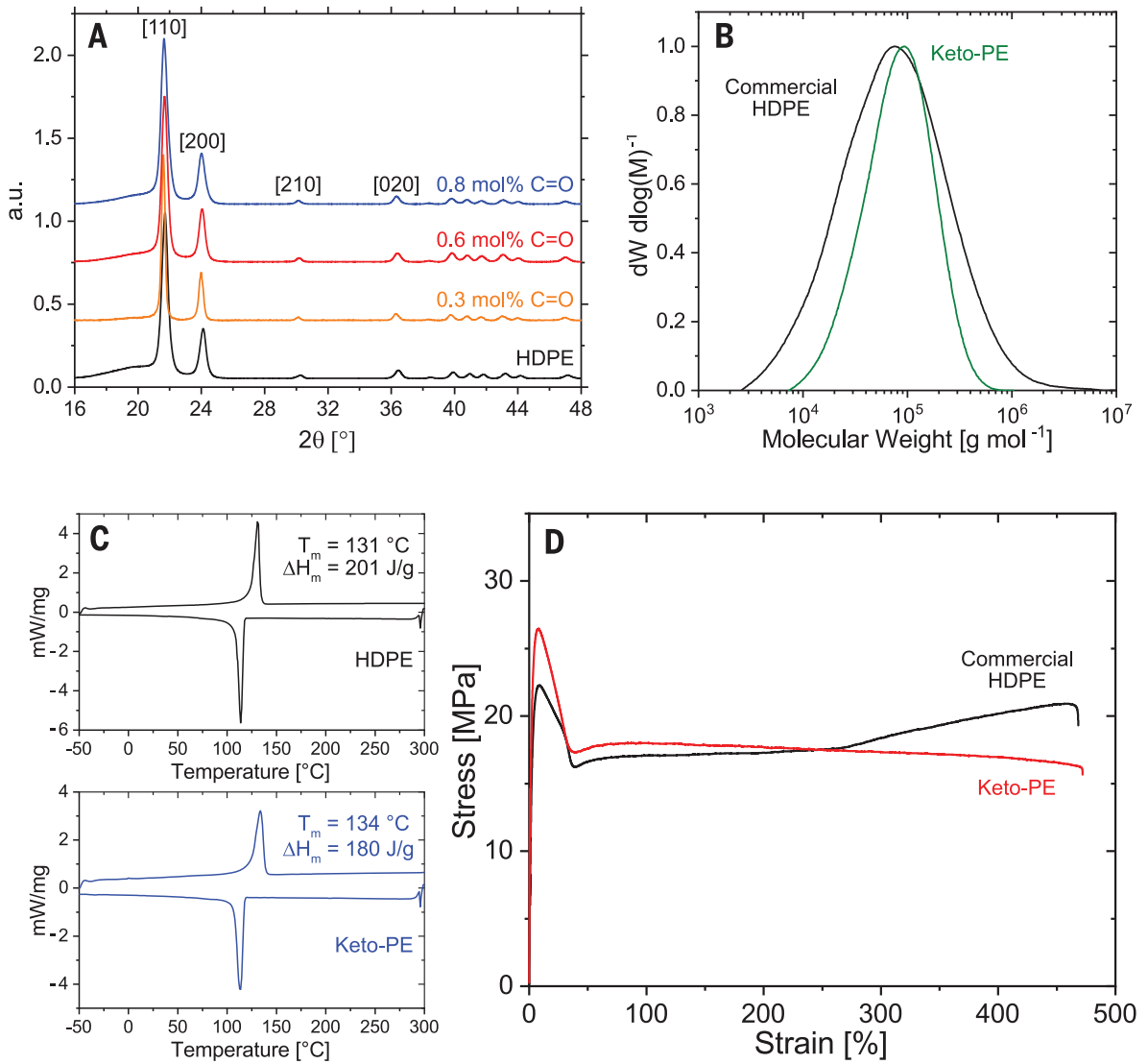

Fig. 3. Materials characterization of copolymers. (A) WAXS diffractograms of copolymers with different keto content and commercial HDPE. Reflections correspond to the orthorhombic unit cell of polyethylene (see supplementary materials for further details). (B) GPC trace of copolymer (1.0 mol\% keto repeat units), and commercial HDPE for comparison. (C) DSC trace of copolymer (0.8 mol\% keto repeat units), and commercial HDPE for comparison. (D) Representative stress-strain curves of injection molded keto-modified polyethylene $(0.6$ mol\% keto repeat units) in comparison to commercial HDPE (see supplementary materials for further data).

This confirms that no undesired additional chain transfer pathways are operative in $\mathrm{CO}$ copolymerizations. Rather, the presence of CO appears to hinder chain transfer and branching as concluded from higher molecular weights and the complete absence of branches in copolymers compared with ethylene homopolymers (Table 1, entries 1 to 4 , versus table S4, entry 2, fig. S17, and figs. S20 to S22), possibly by blocking of coordination sites for $ß$-hydride transfer by CO.

Access to in-chain functionalized polyethylene, which possess the desirable material properties of HDPE, offers perspectives for polyolefins that are less persistent in the environment in enabling photolytic and oxidative chain breakdown. The keto contents reported are reasonable in the sense that the lengths of the polyethylene segments between the installed possible break points correspond to alkane lengths that can be mineralized $(33,34)$. Beyond this rough consideration, the mechanisms and rates of degradation will depend strongly on specific environmental conditions, and in this context an understanding of desirable profiles of the long-term behavior of nonpersistent materials remains to be established (35).

\section{REFERENCES AND NOTES} (2016).

2. G. R. Strobl, The Physics of Polymers: Concepts for 2007).

3. T. O. Morgen, M. Baur, I. Göttker-Schnetmann, S. Mecking, Nat. Commun. 11, 3693 (2020).

4. G. H. Hartley, J. E. Guillet, Macromolecules 1, 165 (1968).

5. A. L. Andrady, J. E. Pegram, Y. Song, J. Environ. Polym. Degrad. 1. 117 (1993)

6. P. Ortmann, F. P. Wimmer, S. Mecking, ACS Macro Lett. 4, 704 (2015)

7. A. Bunescu, S. Lee, Q. Li, J. F. Hartwig, ACS Cent. Sci. 3, 895 (2017).

8. L. Chen et al., Chem 7, 137 (2021).

9. F. C. Rix, M. Brookhart, P. S. White, J. Am. Chem. Soc. 118 , 4746 (1996)

10. E. Drent, P. H. M. Budzelaar, Chem. Rev. 96, 663 (1996)

11. A. Piovano, J. Zarupski, E. Groppo, J. Phys. Chem. Lett. 11 , $5632(2020)$
1. M. Stürzel, S. Mihan, R. Mülhaupt, Chem. Rev. 116, 1398 Understanding their Structures and Behavior, (Springer, ed. 3 ,
12. W. Bertleff, M. Roeper, X. Sava, in Ullmann's Encyclopedia of Industrial Chemistry (Wiley, 2012), pp. 73-97.

13. Press release by Hyosung of May 5, 2016: Hyosung, introduces the polyketone brand 'POKETONE'T' to the global market (retrieved June 8, 2021 from www.poketone.com).

14. Z. Chen, M. Brookhart, Acc. Chem. Res. 51, 1831 (2018)

15. C. Tan, C. Chen, Angew. Chem. Int. Ed. 58, 7192 (2019)

16. A. Keyes et al., Angew. Chem. Int. Ed. 58, 12370 (2019).

17. A. Nakamura, S. Ito, K. Nozaki, Chem. Rev. 109, 5215 (2009).

18. E. Drent, R. van Dijk, R. van Ginkel, B. van Oort, R. I. Pugh, Chem. Commun. 9, 964 (2002).

19. C. Chen et al., Organometallics 30, 5248 (2011).

20. L. Bettucci et al., Dalton Trans. 2007, 5590 (2007).

21. D. K. Newsham, S. Borkar, A. Sen, D. M. Conner, B. Goodall, Organometallics 26, 3636 (2007)

22. S. S. Soomro, D. Cozzula, W. Leitner, H. Vogt, T. E. Müller, Polym. Chem. 5, 3831 (2014).

23. S. Mecking, M. Schnitte, Acc. Chem. Res. 53, 2738 (2020).

24. W. Keim, Angew. Chem. Int. Ed. 52, 12492 (2013).

25. K. A. Ostoja Starzewski, J. Witte, Angew. Chem. Int. Ed. 26, 63 (1987).

26. U. Klabunde, S. D. Ittel, J. Mol. Catal. 41, 123 (1987)

27. W. Keim, F. H. Kowaldt, R. Goddard, C. Krueger, Angew. Chem. Int. Ed. 17, 466 (1978).

28. B. S. Xin et al., J. Am. Chem. Soc. 139, 3611 (2017).

29. Y. Zhang, H. Mu, L. Pan, X. Wang, Y. Li, ACS Catal. 8, 5963 (2018).

30. C. Stafford, B. A. Arndtsen, Inorg. Chim. Acta 369, 231 (2011).

31. D. Jeremic, in Ullmann's Encyclopedia of Industrial Chemistry; Wiley, 2014), pp. 1-42.

32. C. E. Ash, D. G. Waters, A. A. Smaardijk, Soc. Plast. Eng. 53, 2319 (1995).

33. J. R. Haines, M. Alexander, J. Appl. Microbiol. 28, 1084 (1974)

34. F. Kawai et al., Polym. Degrad. Stabil. 86, 105 (2004).

35. M. T. Zumstein, R. Narayan, H. E. Kohler, K. McNeill, M. Sander, Environ. Sci. Technol. 53, 9967 (2019).

\section{ACKNOWLEDGMENTS}

We thank I. Göttker-Schnetmann for support with NMR data interpretation, N. Mast, M. Eck and M. Häußler for support with polymer synthesis and processing. L. Bolk for DSC and GPC measurements and R. Kirsten for technical support. We further thank D. Dietrich, C. Hauck, and A. Marx and their coworkers for enabling safe laboratory operations by providing rapid COVID testing. Funding: The ERC (Advanced Grant DEEPCAT, 832480) is gratefully acknowledged. F.L. thanks the Alexander von Humboldt-Foundation for a postdoctoral research stipend. Author contributions: S.M. conceived the concept. M.B., F.L., T.M., L.O., and S.M. jointly devised the experimental programm. F.L., M.B., and T.M. synthesized and characterized catalyst precursors. M.B. and T.M. performed polymerization experiments with phosphinophenolato catalysts. L.O. performed experiments with salicylaldiminato catalysts. M.B. and T.M performed microstructure analysis and experiments on materials properties. All authors jointly wrote the manuscript. Competing interests: The authors declare no competing interests. 